BMJ Open

Respiratory

Research

\title{
Comprehensive and long-term surveys of COVID-19 sequelae in Japan, an ambidirectional multicentre cohort study: study protocol
}

Kensuke Nakagawara, ${ }^{1}$ Ho Namkoong ${ }^{2}$ Hideki Terai, ${ }^{1}$ Katsunori Masaki (D) , ${ }^{1}$ Takae Tanosaki, ${ }^{1}$ Kyoko Shimamoto, ${ }^{3}$ Ho Lee, ${ }^{1}$ Hiromu Tanaka, ${ }^{1}$ Satoshi Okamori, ${ }^{1}$ Hiroki Kabata, ${ }^{1}$ Shotaro Chubachi, ${ }^{1}$ Shinnosuke Ikemura, ${ }^{1}$ Hirofumi Kamata, ${ }^{1}$ Hiroyuki Yasuda, ${ }^{1}$ Ichiro Kawada, ${ }^{1}$ Makoto Ishii, ${ }^{1}$ Yoshiki Ishibashi, ${ }^{4}$ Sei Harada, ${ }^{4}$ Takanori Fujita, ${ }^{5}$ Daisuke Ito, ${ }^{6}$ Shogyoku Bun, ${ }^{7}$ Hajime Tabuchi, ${ }^{7}$ Sho Kanzaki, ${ }^{8}$ Eisuke Shimizu, ${ }^{9}$ Keitaro Fukuda, ${ }^{10}$ Jun Yamagami, ${ }^{10}$ Keigo Kobayashi, ${ }^{11}$ Toshiyuki Hirano, ${ }^{11}$ Takashi Inoue, ${ }^{11}$ Junko Kagyo, ${ }^{12}$ Tetsuya Shiomi, ${ }^{12}$ Keiko Ohgino, ${ }^{13}$ Koichi Sayama, ${ }^{13}$ Kengo Otsuka, ${ }^{14}$ Naoki Miyao, ${ }^{14}$ Toshio Odani, ${ }^{15}$ Yoshitaka Oyamada, ${ }^{16}$ Keita Masuzawa, ${ }^{17}$ Sohei Nakayama, ${ }^{17}$ Yusuke Suzuki, ${ }^{17}$ Rie Baba, ${ }^{18}$ Ichiro Nakachi, ${ }^{18}$ Naota Kuwahara, ${ }^{19}$ Takashi Ishiguro, ${ }^{20}$ Shuko Mashimo, ${ }^{21}$ Naoto Minematsu, ${ }^{22}$ Soichiro Ueda, ${ }^{23}$ Tadashi Manabe, ${ }^{24}$ Yohei Funatsu, ${ }^{24}$ Hidefumi Koh, ${ }^{24}$ Takashi Yoshiyama, ${ }^{25}$ Fumitake Saito, ${ }^{26}$ Kota Ishioka, ${ }^{27}$ Saeko Takahashi, ${ }^{27}$ Morio Nakamura, ${ }^{27}$ Ai Goto, ${ }^{28}$ Norihiro Harada (1) , ${ }^{28}$ Yu Kusaka (1) , ${ }^{29}$ Yasushi Nakano, ${ }^{30}$ Kazumi Nishio, ${ }^{30}$ Hiroki Tateno, ${ }^{31}$ Ryuya Edahiro, ${ }^{32}$ Yoshito Takeda, ${ }^{32}$ Atsushi Kumanogoh, ${ }^{32}$ Nobuhiro Kodama, ${ }^{33}$ Masaki Okamoto, ${ }^{34}$ Akira Umeda, ${ }^{35}$ Kazuto Hagimura, ${ }^{36}$ Toshiro Sato, ${ }^{37}$ Naoki Miyazaki, ${ }^{38}$ Ryo Takemura, ${ }^{38}$ Yasunori Sato, ${ }^{38}$ Toru Takebayashi, ${ }^{4}$ Jin Nakahara, ${ }^{6}$ Masaru Mimura, ${ }^{7}$ Kaoru Ogawa, ${ }^{8}$ Shigeto Shimmura, ${ }^{9}$ Kazuno Negishi, ${ }^{9}$ Kazuo Tsubota, ${ }^{9}$ Masayuki Amagai, ${ }^{10}$ Rei Goto, ${ }^{39}$ Yoko Ibuka, ${ }^{40}$ Naoki Hasegawa, ${ }^{2}$ Yuko Kitagawa, ${ }^{41}$ Takanori Kanai, ${ }^{42}$ Koichi Fukunaga ${ }^{1}$

To cite: Nakagawara $\mathrm{K}$, Namkoong $\mathrm{H}$, Terai $\mathrm{H}$, et al. Comprehensive and long-term surveys of COVID-19 sequelae in Japan, an ambidirectional multicentre cohort study: study protocol. BMJ Open Resp Res 2021;8:e001015. doi:10.1136/ bmjresp-2021-001015

Received 10 June 2021 Accepted 2 November 2021

Check for updates

(C) Author(s) (or their employer(s)) 2021. Re-use permitted under CC BY-NC. No commercial re-use. See rights and permissions. Published by BMJ.

For numbered affiliations see end of article.

Correspondence to Dr Ho Namkoong; hounamugun@gmail.com

\section{ABSTRACT}

Introduction The rapid spread of COVID-19 posed a global burden. Substantial number of people died of the disease in the acute phase of infection. In addition, a significant proportion of patients have been reported to suffer from post-acute phase symptoms, sequelae of COVID-19, which may negatively influence the quality of daily living and/or socioeconomic circumstances of the patients. However, no previous study has comprehensively and objectively assessed the quality of life of patients by using existing international scales. Further, evidence of socioeconomic consequences among patients with COVID-19 is scarce. To address the multidimensional issues from sequelae of COVID-19, evidence from comprehensive surveys beyond clinical perspectives is critical that investigates health, and social determinants of disease progression as well as socioeconomic consequences at a large scale.

Methods and analysis In this study, we plan to conduct a nationwide and comprehensive survey for the sequelae of COVID-19 in a total of 1000 patients diagnosed at 27 hospitals throughout Japan. This study will evaluate not only the health-related status of patients from clinical perspectives but also the Health- related Quality of Life (HRQDL) scores, socioeconomic status and consequences to discuss the sequelae of the disease and the related risk factors. The primary endpoint is the frequency of long-term complications of COVID-19 infection. The secondary endpoints are risk factors for progression to sequelae of COVID-19 infection. The study will provide robust and important evidence as a resource to tackle the issues from the sequelae of COVID-19 from the multi-dimensional perspectives.

Ethics and dissemination This trial was approved by the Keio University School of Medicine Ethics Committee (20200243, UMIN000042299). The results of this study will be reported at a society meeting or published in a peer-reviewed journal.

\section{INTRODUCTION}

In early 2020, an outbreak of SARS-CoV-2 infection also known as COVID-19 spread around the world. In Japan, the outbreak began in March 2020, and by November, the total number of infected people had reached 100,000. The pandemic imposed major 
limitations in the lives of many patients. SARS-CoV-2 infection may cause a wide variety of symptoms, including diarrhoea and olfactory and taste disturbances, in addition to respiratory symptoms such as cough, sore throat and fever. ${ }^{1}$ According to the study by the WHO using data from China, the first country in the world to experience a COVID-19 pandemic, mild cases of COVID-19 infection improve in about 2 weeks and severe cases in 3-6 weeks. ${ }^{2}$

However, the sequelae of COVID-19 have been recently attracting attention, and many patients who were discharged from the hospital or returned to their pre-disease lives after recovery of their general condition have been found to show sequelae and have not completely recovered to their pre-disease health. In a study in the USA, ${ }^{3} 60 \%$ of patients complained of some aftereffects at 3 weeks after COVID-19 diagnosis, and in an Italian study, ${ }^{4}$ about $90 \%$ of patients were still dissatisfied with their health 2 months after the onset of the disease. ${ }^{4}$ Many of these patients complained of symptoms such as fatigue, dyspnoea and joint pain for a long time. ${ }^{45}$ Some patients have suffered economic and social losses due to physical and mental disabilities that interfere with their work. In a Chinese survey, ${ }^{5}$ sequelae were more varied and included alopecia, psychiatric symptoms and cardiovascular-related symptoms, and many patients' social lives are greatly threatened by these aftereffects. ${ }^{6}$ Thus, various sequelae of COVID-19 have been reported globally and are attracting much attention from a clinical perspective and beyond.

Additionally, the previous reports indicated that the incidence of olfactory taste disorder in Asians was around $18 \%$ and the incidence of olfactory dysgeusia in Caucasians was higher than in Asians. ${ }^{7}$ Therefore, there may be racial differences in the symptoms, frequency and severity of sequelae. In Japan, a study of 78 patients at the National Center for Global Health and Medicine followed the course of various symptoms (fever, cough, fatigue, dyspnoea, dysgeusia, dyssomnias, sputum production and chest pain) after discharge from the hospital by telephone interviews. ${ }^{8}$ However, this was a single-centre survey with a small number of patients that focused on clinical symptoms and was limited to urban areas. Thus, there have been no comprehensive, long-term large surveys of the COVID-19 sequelae in Japan. Globally, the occurrence and duration of COVID-19 sequelae are largely unknown, and the long-term complications caused by COVID-19 have not been evaluated.

Besides these significant clinical burdens that patients with COVID-19 carry, existing evidence also suggests the socioeconomic influence of COVID-19 against these patients. ${ }^{9}$

For example, the severity and mortality of COVID-19 are known to vary with comorbidity and age, ${ }^{10}$ but the incidence and duration of sequelae may vary in each case. In addition, COVID-19 mortality is reported to be affected by the economic status of the region where the disease occurs. ${ }^{11}$ The incidence and duration of the clinical sequelae of COVID-19 may finally vary by socioeconomic circumstance, and the sequalae may have distinct socioeconomic consequences among these patients. Yet, few studies have evaluated the association between social background, economic status, health-related quality of life and sequelae in patients with COVID-19 to understand the socioeconomic impact of COVID-19 on the patients' life.

This study is a comprehensive multicentre survey of Japanese patients with COVID-19 that used a questionnaire to investigate the occurrence of sequelae, quality of life (QoL) and socioeconomic changes after the onset of COVID-19, and evaluated the long-term impact of the COVID-19 pandemic on Japanese patients as well as on Japanese society. All hospitalised patients with COVID-19 at the participating hospitals throughout Japan including both community hospitals and university hospitals are included in the survey, which reduces selection bias and allows for accurate assessment of the prevalence of each symptom. By comparing the results of this study with those of other studies conducted abroad, we can compare the differences in the occurrence of sequelae between races and populations. This study also allows comparison of the severity at the acute phase and the occurrence of sequelae at the chronic phase and evaluation of the correlation. This study also assesses socioeconomic circumstances and consequences among patients with COVID-19 to understand the multidimensional issues from sequelae of COVID-19. Elucidation of the complications and sequelae of COVID-19 in Japan from multidisciplinary perspectives will provide useful information for both patients and society to appropriately confront COVID-19 in the future.

\section{METHODS AND ANALYSIS \\ Study design}

This study is designed as a multicentre, ambidirectional cohort study (planned to run from November 2020 to March 2022) conducted using existing international quality of life assessment scale and original questionnaires. Approximately 1000 patients from 27 medical facilities will participate in this study in Japan. These participants have been registered from facilities across the country including urban, suburban and rural area. The inclusion criteria are as follows: age over 18 years, agreement to participate this survey, diagnosed with COVID-19 by a SARS-CoV-2 PCR test or an antigen test, admitted to the participating medical centres. The exclusion criteria are age under 18 years or patients with language impairment, cognitive impairment or psychiatric disorders that interfere with understanding of informed consent or reading and answering the questionnaires in this study.

\section{Study objectives}

The primary objective was to investigate the frequency and duration of long-term COVID-19 sequelae in Japan. The secondary objectives were to (1) identify the risk 
Expected registration cases $=1,000$ cases

Patients diagnosed with COVID-19 and discharged from the hospital after treatment at the participating hospitals.
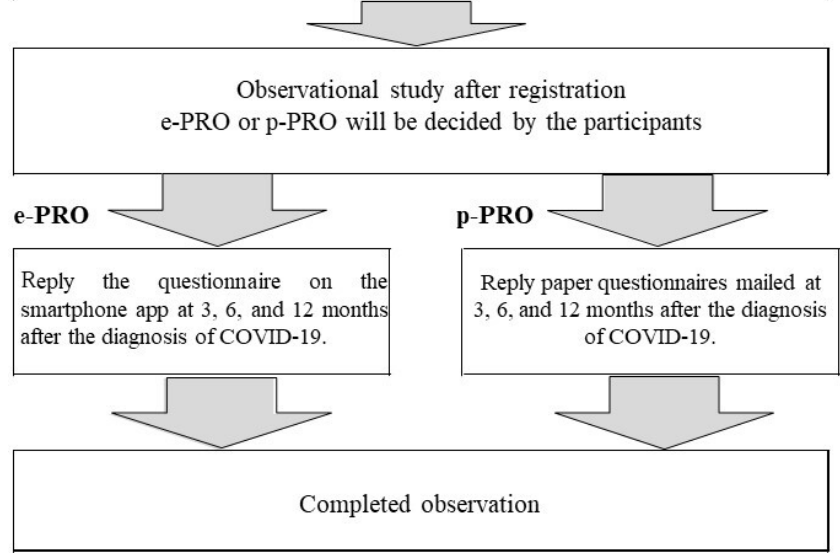

Figure 1 After confirming the research collaborator's consent to participate and cooperate in this study, basic and clinical information of the research collaborator, such as treatment history, will be registered in the case information registration system, and the electronic patientreported outcome (e-PRO) or paper patient-reported outcome ( $p-P R O)$ will be set up. Participants can choose between e-PRO or $\mathrm{p}-\mathrm{PRO}$ of their own initiative. The principal investigator will inform the research collaborators of the details of the e-PRO setup by a smartphone app or notification of $\mathrm{p}-\mathrm{PRO}$ by mail, and collect information regarding sequelae at 3, 6 and 12 months after COVID-19 diagnosis by performing a questionnaire survey.

factors for development of sequelae the relevance of sequelae to acute severity of COVID-19, (2) investigate the influence of COVID-19 sequelae on health-related quality of life and mental health, (3) the association of COVID-19 sequelae with socioeconomic circumstances and consequences, and (4) evaluation of rehabilitation for COVID-19 aftereffects.

All of the QoL rating scales are international standards, it will be possible to compare them in various ways with previous studies or studies to be reported in the future.

\section{Research schedule}

The participating institutions will send a participant information sheet to the potential participants, informing them about the details of the study and enquiring about their willingness to participate in the study. After the participant's consent has been obtained, patient information will be registered in the case information registration system, and electronic patient-reported outcome (e-PRO) or paper patient-reported outcome (p-PRO) will be set up. The study flow is shown in figure 1. At baseline, the principal investigator will collect clinical information, and the participants will also be asked to complete e-PRO or p-PRO form at 3, 6 and 12 months after the date of COVID-19 diagnosis.
Table 1 Symptoms assessed in the first questionnaire

\begin{tabular}{|c|c|c|}
\hline Fever & Cough & Sputum \\
\hline Poor concentration & Fatigue & Alopecia \\
\hline Memory impairment & Headache & Sore throat \\
\hline Muscular weakness & Unconsciousness & Diarrhoea \\
\hline Abdominal pain & Numbness & Myalgia \\
\hline $\begin{array}{l}\text { Eye-related } \\
\text { symptoms }\end{array}$ & Dyspnoea & Arthralgia \\
\hline $\begin{array}{l}\text { Hypersensitivity to } \\
\text { sound, light and } \\
\text { smell }\end{array}$ & Tinnitus & Rash \\
\hline Sleeping disorders & Olfactory disorders & $\begin{array}{l}\text { Taste } \\
\text { disorders }\end{array}$ \\
\hline
\end{tabular}

\section{Information on the questionnaire}

The questionnaire is divided into two main sections. The first section comprehensively surveys the symptoms that appeared at the time of COVID-19 diagnosis, their duration, timing of appearance, severity and sequelae. This section is the original questionnaire developed by this study team and the symptoms evaluated in this survey are summarised in table 1. For the duration of each symptom, there are seven periods to choose from less than 1 week, 1 week to 1 month, 1-3 months, 3-6 months, 6-12 months, more than 12 months, and the symptom still persists. In particular, patients with respiratory, visual, olfactory, gustatory and memory disturbances are assessed using a more detailed questionnaire. Responders with sequelae of dyspnoea will be assessed in detail by the Modified Medical Research Council (mMRC) Dyspnea Scale. ${ }^{12}$ The additional questionnaire on olfactory and taste disorders will be used, which is developed by American Academy of Otolaryngology-Head and Neck Surgery. ${ }^{13}$ Similarly, additional questions regarding memory loss correspond to the Everyday Memory Checklist Japanese edition. ${ }^{14}$ We also will evaluate symptoms that had improved and re-emerged after the onset of COVID-19, as well as new symptoms that appeared after discharge.

In the second part of the questionnaire, the patients' quality of life will be assessed by international rating scales, including the HRQoL scales and other related tools on socioeconomic aspects. In this survey, we will be using Short Form-8 (SF-8), ${ }^{15}$ Euro Quality of Life 5 Dimensions 5 Level (EQ-5D-5L), ${ }^{16}$ Hospital Anxiety and Depression Scale (HADS), ${ }^{17}$ the Pittsburgh Sleep Quality Index, ${ }^{18}$ WHO Health and Work Performance Questionnaire (short form) Japanese edition, ${ }^{19}$ Fear of Coronavirus-19 Scale $^{20}$ and the Performance status. ${ }^{21}$ We will also assess the participants' socioeconomic circumstances and relevant changes in the COVID-19 pandemic, including the situation of employment, income, social life and informal care, in this section. To evaluate the onset and clinical course of the sequelae, participants will be asked to reflect on their condition on 3, 6 and 12 months after 
the onset of COVID-19 infection, and responses will be collected for each point in time.

\section{Collection of clinical information}

At the time of participant enrolment, the principal investigator will collect clinical information from medical records, including participants' symptoms and clinical information such as age, sex, comorbidity, physical and clinical examination findings laboratory findings, ECG, radiological findings and clinical course. Subsequently, information on the sequelae of COVID-19 infection will be collected at 3, 6 and 12 months after COVID-19

Table 2 Study calendar

\begin{tabular}{|c|c|c|c|c|}
\hline & At registration & $\begin{array}{l}3 \text { months after } \\
\text { diagnosis }\end{array}$ & $\begin{array}{l}6 \text { months after } \\
\text { diagnosis }\end{array}$ & $\begin{array}{l}12 \text { months after } \\
\text { diagnosis }\end{array}$ \\
\hline \multicolumn{5}{|l|}{ Participant background } \\
\hline Lifestyle and preference history & $\bigcirc$ & & & \\
\hline $\begin{array}{l}\text { Medical history: date of diagnosis, } \\
\text { comorbidities, allergies, medications, } \\
\text { pregnancy status and route of infection }\end{array}$ & O & & & \\
\hline COVID-19 severity & $\bigcirc$ & & & \\
\hline \multicolumn{5}{|l|}{ Physical findings } \\
\hline Vital signs & $\bigcirc$ & & & \\
\hline Height and weight & O & & & \\
\hline Clinical symptoms* & $\bigcirc$ & $\bigcirc$ & $\mathrm{O}$ & $\mathrm{O}$ \\
\hline \multicolumn{5}{|l|}{ Clinical laboratory data } \\
\hline Blood test findings $\dagger$ & O & & & \\
\hline ECG & $\bigcirc$ & & & \\
\hline \multicolumn{5}{|l|}{ Radiological examination } \\
\hline Chest radiography & 0 & & & \\
\hline Chest CT scan & O & & & \\
\hline Clinical course & O & & & \\
\hline Treatment progress and outcomesł & $\bigcirc$ & & & \\
\hline Rehabilitation survey & $\bigcirc$ & 0 & $\mathrm{O}$ & $\mathrm{O}$ \\
\hline \multicolumn{5}{|l|}{ e-PRO or $p-P R O$} \\
\hline SF-8 Standard Ver. & & 0 & $\mathrm{O}$ & $\mathrm{O}$ \\
\hline EQ-5D-5L & & $\bigcirc$ & $\mathrm{O}$ & $\mathrm{O}$ \\
\hline HADS & & 0 & O & $\mathrm{O}$ \\
\hline The Pittsburgh Sleep Quality Index & & 0 & $\mathrm{O}$ & $\mathrm{O}$ \\
\hline $\begin{array}{l}\text { WHO Health and Work Performance } \\
\text { Questionnaire (short form) Japanese edition }\end{array}$ & & $\bigcirc$ & $\mathrm{O}$ & $\mathrm{O}$ \\
\hline Fear of Coronavirus-19 Scale, & & 0 & $\mathrm{O}$ & $\mathrm{O}$ \\
\hline $\begin{array}{l}\text { Modified Medical Research Council } \\
\text { Dyspnea Scale }\end{array}$ & & $\bigcirc$ & $\mathrm{O}$ & $\mathrm{O}$ \\
\hline $\begin{array}{l}\text { Investigation of sequelae and symptoms other } \\
\text { than those covered in the above assessments }\end{array}$ & & $\bigcirc$ & O & 0 \\
\hline Questionnaire about economic status & 0 & & & \\
\hline
\end{tabular}

*Fever, cough, sputum, sore throat, nasal congestion, dyspnoea, diarrhoea, vomiting, fatigue, olfactory disturbances, taste disorders, etc. †Red cell count, white cell counts, haemoglobin level, hematocrit, platelet count, total protein, albumin, total bilirubin, AST, ALT, LDH, ALP, BUN, creatinine, $\mathrm{Na}, \mathrm{K}, \mathrm{Cl}, \mathrm{Ca}$, IP, blood sugar, HbA1c, total cholesterol, LDL cholesterol, HDL cholesterol, triglyceride, ferritin, KL-6, and BNP levels, urinary pneumococcal antigen, urinary Legionella antigen, PCT, etc.

fUse of oxygen and ventilators, antibiotics and antivirals, steroids, treatment effects, date of diagnosis, date of admission and discharge, etc.

ALP, alkaline phosphatase; ALT, alanine aminotransferase; AST, aspartate aminotransferase; BNP, brain natriuretic peptide; BUN, blood urea nitrogen; e-PRO, electronic patient-reported outcome; EQ-5D-5L, Euro Quality of Life 5 Dimensions 5 Level; HADS, Hospital Anxiety and Depression Scale; HbA1c, Hemoglobin A1c; HDL, high-density lipoprotein cholesterol; IP, inorganic phosphorus; LDH, lactate dehydrogenase ; LDL, low-density lipoprotein cholesterol; p-PRO, paper patient-reported outcome; SF-8, Short Form-8. 
diagnosis with e-PRO or p-PRO (table 2). The participants can choose between e-PRO or p-PRO at the start. From this survey, the specific time of appearance and disappearance of the sequelae, as well as the patient's background, will be determined to gain an overall understanding of the sequelae of COVID-19 infection in Japan. In addition, using health-related quality of life questionnaires (SF-8, Eq-5D-5L, HADS, the Pittsburgh Sleep Quality Index, WHO Health and Work Performance Questionnaire (short form) Japanese edition, Fear of Coronavirus-19 Scale and mMRC Dyspnea Scale, we will evaluate not only the disability caused by physical sequelae such as respiratory symptoms in daily life but also the psychological anxiety caused by these sequelae. We will also research the economic status of the subjects and assess association with COVID-19 infection. In this study, we do not have a clear protocol for rehabilitation. We will try to question patients about the rehabilitation they received after discharge from the acute care hospital, to the extent possible.

SARS-CoV-2 can be transmitted to others if the virus released in respiratory secretions when an infected person coughs, sneezes or speaks is inhaled or comes in direct contact with mucous membranes. ${ }^{22}$ Contact with contaminated materials is also not considered a major route of transmission, but it is possible to become infected if your hands become contaminated with these secretions or if you touch your eyes, nose or mouth after touching a contaminated surface. ${ }^{22}$ We believe that the electronic medium, e-PRO, to collect the sequelae information in this study, is also effective in preventing the transmission of SARS-CoV-2 from subjects to researchers. In addition, E-PRO is considered to be an effective data collection method because of its various advantages such as prevention of erroneous entries, speedy collection of questionnaires, abatement of the burden on participants and reduction of the cost to send questionnaires.

All data will be collected by the Data Management Centre at $3 \mathrm{H}$ Holdings (Tokyo, Japan). There will be no direct communication between investigators and data managers. The clinical data entry, coding, data management and reporting will be performed using the data management system 3H-P Guardian (3H Holdings).

\section{Sample size calculations and statistical analysis}

According to a survey conducted by the National Institute of Infectious Diseases in Japan, as of the end of August 2020, there were approximately 40000 cases of new coronavirus infection and less than 1000 deaths in Japan. It is reported that about 8000 patients required inpatient treatment for COVID-19, and about 7000 patients were discharged or released from hospital. ${ }^{23}$ Based on the current number of patients, we estimate that the number of patients who can be tracked and registered at the research cooperating facilities will be about $30 \%$ of the patients who received inpatient treatment, and eventually 1000 patients will be eligible for registration.
For the primary outcomes, summary statistics such as frequency and mean, SD will be described for each sequela at each time point. An exploratory analysis of differences by severity will be conducted using logistic regression or Mantel-Haenszel test. Transition in values will be presented over time, and exploratory analysis will be conducted by analysis of variance (ANOVA).

For secondary outcomes, summary statistics will be described for each QoL-related rating scale, clinical findings, laboratory examinations and actual rehabilitation status for each case at each time point. We will also conduct exploratory analysis of various scores by severity of illness using ANOVA and generalised linear models. In addition, factors that predict other sequelae, such as pulmonary dysfunction and decreased QoL, will be explored by multivariate analysis.

Further statistical analyses will be conducted to assess the association of COVID-19 sequelae with socioeconomic circumstances and consequences, employing multivariate analysis and structural equation modelling.

\section{Confidentiality and data retention}

Responses collected in this questionnaire will be kept strictly anonymous and confidential and will not be used outside of this study to identify the participants. No participant identifiers will be included in the case report form. Participants will be anonymised in both the e-PRO and p-PRO media using numbers generated by the research facility. The anonymised list will be kept securely locked by the personal information manager. The personal information of the participants will be kept confidential, even in the event of allowing access to the original data of the study or publication in a medical journal. For answers by e-PRO, in addition to the anonymisation number, a different ID and password will be used for login authentication to the server. The password is set individually by the participant, and the e-PRO data on the server do not contain any personal information.

\section{Ethics and dissemination}

We have received approval from the research ethics committees of the Keio University School of Medicine, JAPAN, to conduct this study (20200243). All respondents to this study will be accredited only if they have completed the informed consent questions in advance and have indicated their consent for participation in the study. Participation in this study is completely voluntary. In addition, participants will be informed that they are free to withdraw their consent at any time. All participants may choose not to respond to one or more specific questions. The UMIN ID for this survey is 000042299.

\section{Patient and public involvement statement}

No patient involved in the development of the research question, study design, recruitment, outcome measures, dissemination plans and conduction of the study. 
The study findings will be disseminated through openaccess scientific publications, stakeholder networks and national/international meetings.

\section{DISCUSSION}

This long-term, large, ambidirectional study aims to comprehensively investigate the various sequalae of COVID-19 and more objectively evaluate the QoL by using multiple international rating scales. There is no precedent for a study of the same scale, and the information from this survey will be very meaningful. Only moderate and severe cases of COVID-19 were included in this study, and mild cases without pneumonia were excluded. Therefore, it is not possible to assess the sequelae of mild cases from this study, as these results may be influenced by the severity of the acute phase.

In this study, we do not have a protocol for rehabilitation. However, in some cases, after completing the acute treatment, the patient was transferred to a chronic hospital for rehabilitation owing to a decrease in the Activities of Daily Living (ADL) and the need for oxygen therapy. We will try to investigate each patient's rehabilitation status-whether they have received rehabilitation programme after discharge from the acute care hospital-via questionnaires.

The first part of the questionnaire investigates the various symptoms listed in table 1 , including respiratory, gastrointestinal and skin symptoms. No previous study has conducted such an extensive and long-term follow-up of COVID-19 sequelae. We will learn more about the clinical course of COVID-19 by assessing the reappearance of symptoms after improvement, their duration and the relationship between sequelae and the severity of illnesses, comorbidities and age groups. In particular, the American Academy of Otorhinolaryngology's International Scale and Everyday Memory Rating Scale are being used to assess olfactory, taste and memory impairments, ensuring external validity and allowing for comparisons between races and nations.

The second part of the questionnaire assesses Japanese patients with COVID-19 from the multidimensional perspectives such as quality of life, mental health and socioeconomic consequences including work performance by using six international standard rating scales, namely, the SF-8, EQ-5D-5L, HADS, the Pittsburgh Sleep Quality Index, the WHO Health and Work Performance Questionnaire (WHO-HPQ) and the Fear of COVID-19 Scale (FCV-19S), as well as additional original questions. No previous study has comprehensively and objectively assessed the quality of life of Japanese patients with COVID-19 by using these international scales or investigated the socioeconomic influence of COVID-19 among the patients. Comparisons of the results obtained with these six scales is expected to yield new insights, especially since no previous study has employed these six scales simultaneously. In addition, all these scales are frequently used internationally, ensuring external validity and allowing comparisons of the results with previous studies.

The risk factors for COVID-19 severity have been actively debated worldwide, and many factors have been elucidated. ${ }^{82}$ A previous study evaluated respiratory function tests at hospital discharge in 110 cases of COVID-19 to determine risk factors for sequelae and suggested that disease severity was significantly associated with the degree of diffusion impairment on respiratory function tests. ${ }^{25}$ In this nationwide study, we aim to perform more comprehensive and longer-term evaluations of post-discharge sequelae and QoL scores and to analyse risk factors for the appearance of sequelae and deterioration of QoL. This study will help clarify the risk factors for sequelae by comparing the results of questionnaire assessments with the medical record information obtained at the time of hospitalisation.

The mMRC Dyspnea Scale, which will be used as a measure of dyspnoea in this study, is primarily used to assess breathlessness in patients with chronic obstructive pulmonary disease and is highly correlated with other measures of health status, ${ }^{26}$ and it can be used to predict relative future risk of death. ${ }^{27}$ Patients with COVID-19 have been reported to experience prolonged shortness of breath as sequelae, showing significantly higher mMRC scores than those before hospitalisation, ${ }^{28}$ and worsening mMRC is associated with a decline in forced expiratory volume 1.0 (FEV1.0) and diffusing capacity for carbon monoxide $\left(\mathrm{DL}_{\mathrm{CO}}\right)$ in actual respiratory function tests. ${ }^{29}$ By using mMRC as a measure of shortness of breath in this study, it was possible to objectively evaluate the relationship between the sequelae of dyspnoea and treatment or severity in the acute phase, and determine whether this symptom had improved or not.

COVID-19 sequelae include not only physical disabilities but also psychological problems such as insomnia and memory impairment. In one study that evaluated the sequelae in 120 patients with COVID-19 4 months after discharge, approximately $30 \%$ of the patients complained of insomnia and forgetfulness. ${ }^{30}$ In the present study, participants who complain of memory impairment will be additionally assessed with the Everyday Memory Checklist Japanese edition. Although the Japanese version of the Everyday Memory Checklist has never been used for memory disorders in patients with COVID-19, this scale has been reported to be reliable and valid in assessing daily memory problems. ${ }^{14}$ In the present study, we believe that the Everyday Memory Checklist can be used for a detailed examination of the extent to which memory impairments as a sequela of COVID-19 affect daily life.

In this study, we have adopted the SF-8 as a measure of participants' QoL. A previous study used SF-36 to prove that respiratory rehabilitation in patients with COVID-19 improves patients' respiratory function as well as their quality of life. ${ }^{31}$ However, no previous studies have evaluated the quality of life of patients with COVID-19 by SF-8. The SF-8 is classified as a comprehensive scale for assessing HRQoL and allows comparison of between patients with different 
diseases. It also provides a continuous measure of HRQoL from sick to healthy people, allowing patients' health status to be compared with that of the general population. The SF-8 also has only eight questions, which allows for quick evaluations and makes it suitable for large surveys. In addition, the SF-8 has national standards, and the health status of the target group can be compared based on the Japanese average (50 points).

The EQ-5D-5L is a scale for assessing HRQoL that is frequently used in medical settings. It is a five-dimensional questionnaire consisting of the following items: 'degree of mobility,' 'personal management,' 'daily activities,' 'pain/ discomfort,' and 'anxiety/stagnation.' The EQ-5D-5L enables calculation of health-related quality of life that reflects the values of Japanese people. Quality-adjusted life years (QALYs), which can be calculated using the EQ-5D-5L, are a common measure of burden of disease and can be used to evaluate the cost-effectiveness of medical treatment. QALYs can be used as a common measure for many diseases, and multiple effects can be evaluated simultaneously, making the results easy to interpret and compare with other diseases. The QALYs calculated in this study can be used to compare the relationship between treatment during hospitalisation for COVID-19 and post-discharge QoL, and the QoL reductions caused by COVID-19 with those caused by other diseases. A previous study assessing the EQ-5D-5L before and after the onset of COVID-19 found a significant decrease in this score with the onset of COVID-19, suggesting that COVID-19 has a negative influence on the QoL and health burden on the economy. ${ }^{32}$ In a previous Iranian study that assessed HR-QoL using EQ-5D-5L in 420 post-discharge patients with COVID19 , female gender, older age ( $>60$ years), higher education, unemployment, Intensive Care Unit (ICU) admission and diabetes status showed a significant decrease in EQ-5D-5L values $(\mathrm{p}<0.05) .{ }^{33}$ As with these previous studies, the present study allows us to assess the extent of the decline in Japanese health-related quality of life after the onset of COVID-19 and the factors associated with the decline in this score. We can also consider the impact of national origin, race and region of onset on health-related quality of life through comparison with other previous studies.

The HADS is a self-administered questionnaire for patients that contains seven questions each about depression and anxiety, with a total of 14 questions to assess the results. Most clinical studies of COVID-19 using the HADS score have involved healthcare workers and the general population, ${ }^{34}{ }^{35}$ and there is a paucity of studies that have assessed psychiatric symptoms such as anxiety and depression in patients with COVID-19. In a HADS-based study of 105 patients with COVID-19 in Italy, ${ }^{36}$ approximately $30 \%$ of the patients still showed morbidity at 1-3 months after COVID-19 improvement. In that study, HADS scores were significantly lower in patients with prolonged clinical symptoms such as apathy, dyspnoea and cognitive impairment as sequelae. The study also found no correlation between acute illness severity and anxiety and depression. Our study participants will be followed for up to 12 months, allowing a more objective assessment of the long-term evolution of psychiatric symptoms. In addition, our study will provide insights into the correlation between HADS score and sequelae and the severity at the acute phase in Japanese participants, and clarify the differences in HADS scores between races and regions by comparing the findings with those obtained in previous studies.

Previous studies have suggested that patients with a pandemic disease may have insomnia, ${ }^{37}$ and a sleep quality assessment by the Pittsburgh Sleep Quality Index in patients with COVID-19 in Wuhan also clearly showed sleep disturbances. ${ }^{38}$ This study revealed a significantly greater incidence of sleeping disorders among elderly patients and those with any chronic comorbidity. Notably, sleep quality in this study was significantly associated with the patients' subjective perception of disease severity, not an objective clinical classification. Our study is also using the Pittsburgh Sleep Quality Index to assess insomnia in patients with COVID-19. The current study of inpatients can accurately assess the association between severity and comorbidity and sleep disorders, as well as the correlation with other physical and mental symptoms. Few studies have evaluated sleep disturbances in patients with COVID-19, and many studies have been conducted with healthcare professionals and the public. ${ }^{39}$ Thus, we believe that this study, which focused on patients with COVID-19, and evaluated their sleep disorders, will be valuable.

The mental health of workers is known to influence labour productivity in the workplace, and impaired mental health can lead to loss of workforce. In this social context, a situation called absenteeism, in which workers are absent from work due to a disease, is an objective fact and easy to comprehend, while the loss of labour force due to a worker's reduced ability to perform work, called presenteeism, is more difficult to evaluate objectively. The latter is considered to be more severe loss of labour force and is a social problem. In response to this problem, the WHO has proposed the WHO-HPQ to provide an early assessment of workers' mental health, and the Japanese version of this rating scale has already been created. ${ }^{40}$ From 2019 to 2020, although the COVID-19 pandemic severely limited economic activity worldwide, ${ }^{41}$ the extent to which the work performance of patients with COVID-19 was affected has not been assessed, and this study uses the WHO-HPQ to examine that aspect as well. This study will be the first investigation that allows for consideration of COVID-19 patients' clinical information in relation to their work performance.

The current study assesses various aspects of the fear of the COVID-19 pandemic in Japanese patients with FCV-19S Japanese version, which consists of seven questions. The FCV$19 \mathrm{~S}$ is in use worldwide, and previous studies with this scale have already been reported in many countries. Many studies have assessed the fear of the COVID-19 pandemic in the public. ${ }^{43}$ On the other hand, no studies have used the FCV$19 S$ in patients with COVID-19, and the present study will provide an insight into the differences in fear scales between the public and patients. In addition, this study will evaluate the psychometric properties of the Japanese version of FCV$19 \mathrm{~S}$ and reveal its associated factors in Japanese patients. The 
findings will also allow assessment of the factors associated with fear scale scores, such as disease severity, specific symptoms, comorbidity and age groups.

At the end of the second part of the questionnaire, the subjects are also asked about their socioeconomic circumstances and relevant changes in the COVID-19 pandemic, including the situation of employment, income, social life and informal care informal care. This is because wealth status and other socioeconomic positions have been reported to affect the incidence and severity of COVID-19, ${ }^{911}$ while evidence is still scarce to understand the socioeconomic consequences among patients with COVID-19. This study includes patients with COVID-19 from remarkably diverse social backgrounds. Thus, the findings of the study will allow us to assess whether socioeconomic conditions may influence the severity and morbidity of COVID-19 in Japan as well, and to what extent the socioeconomic background of the patients may affect work performance, health-related quality of life and other parts of the patients' life. Further, this study will contribute to fill the global evidence gap of the socioeconomic consequences among patients with COVID-19.

This is the first study in Japan to comprehensively investigate the clinical and socioeconomic information of patients with COVID-19 over a large scale and a long period of time. The results of this study are expected to provide a more accurate clinical picture of COVID-19, including its sequelae using a multidisciplinary approach. Several international rating scales are being used to assess the sequelae, which will allow objective considerations, as well as evaluations of differences between countries and regions and comparisons with previous studies. In particular, studies that have incorporated clinical aspects and socioeconomic consequences including informal care, work performance and other social life environments of patients with COVID-19 have not been reported in the past, and new insights are expected to be gained in this survey.

\section{Author affiliations}

${ }^{1}$ Division of Pulmonary Medicine, Department of Medicine, Keio University School of Medicine, Tokyo, Japan

${ }^{2}$ Department of Infectious Diseases, Keio University School of Medicine, Tokyo, Japan

${ }^{3}$ Keio Global Research Institute, Keio University, Tokyo, Japan

${ }^{4}$ Department of Preventive Medicine and Public Health, Keio University School of Medicine, Tokyo, Japan

${ }^{5}$ Department of Health Policy and Management, Keio University School of Medicine, Tokyo, Japan

${ }^{6}$ Department of Neurology, Keio University School of Medicine, Tokyo, Japan

${ }^{7}$ Department of Neuropsychiatry, Keio University School of Medicine, Tokyo, Japan

${ }^{8}$ Department of Otolaryngology, Keio University School of Medicine, Tokyo, Japan

${ }^{9}$ Department of Ophthalmology, Keio University School of Medicine, Tokyo, Japan

${ }^{10}$ Department of Dermatology, Keio University School of Medicine, Tokyo, Japan

${ }^{11}$ Department of Internal Medicine, Sano Kosei General Hospital, Tochigi, Japan

${ }^{12}$ Department of Internal Medicine, Keiyu Hospital, Kanagawa, Japan

${ }^{13}$ Division of Pulmonary Medicine, Kawasaki Municipal Hospital, Kanagawa, Japan

${ }^{14}$ Department of Internal Medicine, Nihon Koukan Hospital, Kanagawa, Japan
${ }^{15}$ Department of Rheumatology, National Hospital Organization Hokkaido Medical Center, Hokkaido, Japan

${ }^{16}$ Department of Respiratory Medicine, National Hospital Organisation Tokyo Medical Center, Tokyo, Japan

${ }^{17}$ Department of Respiratory Medicine, Kitasato University Kitasato Institute Hospital, Tokyo, Japan

${ }^{18}$ Pulmonary Division, Department Internal Medicine, Saiseikai Utsunomiya Hospital, Tochigi, Japan

${ }^{19}$ Department of Medicine, Division of Allergology and Respiratory Medicine, Showa University Koto Toyosu Hospital, Tokyo, Japan

${ }^{20}$ Department of Respiratory Medicine, Saitama Cardiovascular and Respiratory Center, Saitama, Japan

${ }^{21}$ Department of Respiratory Medicine, Toyohashi Municipal Hospital, Aichi, Japan

${ }^{22}$ Department of Internal Medicine, Hino Municipal Hospital, Tokyo, Japan

${ }^{23}$ Department of Internal Medicine, Saitama Medical Center, Saitama, Japan

${ }^{24}$ Division of Pulmonary Medicine, Department of Internal Medicine,

Tachikawa Hospital, Tokyo, Japan

${ }^{25}$ Respiratory Disease Center, Fukujuji Hospital,Japan Anti-Tuberculosis Association, Tokyo, Japan

${ }^{26}$ Department of Pulmonary Medicine, Eiju General Hospital, Tokyo, Japan

${ }^{27}$ Department of Pulmonary Medicine, Saiseikai Central Hospital, Tokyo, Japan

${ }^{28}$ Department of Respiratory Medicine, Juntendo University Faculty of

Medicine and Graduate School of Medicine, Tokyo, Japan

${ }^{29}$ Department of Respiratory Medicine, Ome Municipal General Hospital, Ome,

Tokyo, Japan

${ }^{30}$ Department of Pulmonary Medicine, Kawasaki Municipal Ida Hospital, Kanagawa, Japan

${ }^{31}$ Department of Pulmonary Medicine, Saitama City Hospital, Saitama, Japan

${ }^{32}$ Department of Respiratory Medicine and Clinical Immunology, Osaka

University Graduate School of Medicine, Osaka, Japan

${ }^{33}$ Department of General Internal Medicine, Fukuoka Tokushukai Hospitali, Fukuoka, Japan

${ }^{34}$ Department of Internal Medicine, Division of Respirology, Neurology, and

Rheumatology, Kurume University School of Medicine, Fukuoka, Japan

${ }^{35}$ Department of Respiratory Medicine, International University of Health and Welfare Shioya Hospital, Tochigi, Japan

${ }^{36}$ Clinical and Translational Research Center, Keio University Hospital, Tokyo, Japan

${ }^{37}$ Department of Organoid Medicine, Keio University School of Medicine, Tokyo, Japan

${ }^{38}$ Biostatistics Unit, Clinical and Translational Research Center, Keio University Hospital, Tokyo, Japan

${ }^{39}$ Graduate School of Business Administration, Keio University, Tokyo, Japan

${ }^{40}$ Faculty of Economics, Keio University, Tokyo, Japan

${ }^{41}$ Department of Surgery, Keio University School of Medicine, Tokyo, Japan

${ }^{42}$ Division of Gastroenterology and Hepatology Department of Internal

Medicine, School of Medicine, Keio University, Tokyo, Japan

Acknowledgements We are thankful to all the patient and public reviewers who took the time to complete the survey and share their experiences with us.

Collaborators Japan COVID-19 Task Force.

Contributors Contributors H Namkoong, H Terai, K Masaki, M Ishii, and K Fukunaga conceived the study and participated in its design and coordination. $\mathrm{H}$ Namkoong, H Terai, K Masaki, M Ishii, and K Fukunaga participated in the design of the study and is responsible for the statistical analysis plan. K Nakagawara, $\mathrm{H}$ Namkoong, H Terai, K Masaki, T Tanosaki, K Shimamoto, S Okamori, H Kabata, S Chubachi, M Ishii, Y Ishibashi, S Harada, T Fujita, D Ito, S Bun, H Tabuchi, S Kanzaki, E Shimizu, K Fukuda, J Yamagami, R Goto, Y lbuka and K Fukunaga participated in the establishment of the study design. The study protocol was developed by all the authors of this manuscript. All authors read and approved the final version of the manuscript.

Funding This study is supported by Health and Labor Sciences Research Grants (20CA2054) and Japan Agency for Medical Research and Development (AMED) (20nk0101611h0002).

\section{Competing interests None declared.}

Patient and public involvement Patients and/or the public were involved in the design, or conduct, or reporting or dissemination plans of this research. Refer to the Methods and analysis section for further details. 
Patient consent for publication Consent obtained from parent(s)/guardian(s) Provenance and peer review Not commissioned; externally peer reviewed. Open access This is an open access article distributed in accordance with the Creative Commons Attribution Non Commercial (CC BY-NC 4.0) license, which permits others to distribute, remix, adapt, build upon this work non-commercially, and license their derivative works on different terms, provided the original work is properly cited, appropriate credit is given, any changes made indicated, and the use is non-commercial. See: http://creativecommons.org/licenses/by-nc/4.0/.

ORCID iDs

Katsunori Masaki http://orcid.org/0000-0003-0909-9409

Norihiro Harada http://orcid.org/0000-0002-0962-5731

Yu Kusaka http://orcid.org/0000-0001-6884-8110

\section{REFERENCES}

1 Stokes EK, Zambrano LD, Anderson KN, et al. Coronavirus Disease 2019 Case Surveillance - United States, January 22-May 30, 2020. MMWR Morb Mortal Wkly Rep 2020;69:759-65.

2 World Health Organization. Director-General's opening remarks at the media briefing on COVID-19, 2020. Available: https://www.who. int/dg/speeches/detail/who-director-general-s-opening-remarks-atthe-media-briefing-on-covid-19-24-february-2020 [Accessed 26 Feb 2020].

3 Tenforde MW, Billig Rose E, Lindsell CJ, et al. Characteristics of Adult Outpatients and Inpatients with COVID-19 - 11 Academic Medical Centers, United States, March-May 2020. MMWR Morb Mortal Wkly Rep 2020;69:841-6.

4 Carfi A, Bernabei R, Landi F, et al. Persistent symptoms in patients after acute COVID-19. JAMA 2020;324:603-5.

5 Xiong Q, Xu M, Li J, et al. Clinical sequelae of COVID-19 survivors in Wuhan, China: a single-centre longitudinal study. Clin Microbiol Infect 2021;27:89-95.

6 van den Borst B, Peters JB, Brink M, et al. Comprehensive health assessment 3 months after recovery from acute coronavirus disease 2019 (COVID-19). Clin Infect Dis 2021;73:e1089-98.

7 von Bartheld CS, Hagen MM, Butowt R. Prevalence of chemosensory dysfunction in COVID-19 patients: a systematic review and meta-analysis reveals significant ethnic differences. ACS Chem Neurosci 2020;11:2944-61.

8 Miyazato Y, Morioka S, Tsuzuki S, et al. Prolonged and late-onset symptoms of coronavirus disease 2019. Open Forum Infect Dis 2020;7:ofaa507.

9 Khalatbari-Soltani S, Cumming RC, Delpierre C, et al. Importance of collecting data on socioeconomic determinants from the early stage of the COVID-19 outbreak onwards. J Epidemiol Community Health 2020;74:620-3.

10 Ishii M, Terai $\mathrm{H}$, Kabata $\mathrm{H}$, et al. Clinical characteristics of 345 patients with coronavirus disease 2019 in Japan: a multicenter retrospective study. J Infect 2020;81:e3-5.

11 Abedi V, Olulana O, Avula V, et al. Racial, economic, and health inequality and COVID-19 infection in the United States. J Racial Ethn Health Disparities 2021;8:732-42.

12 Ferris BG, Project ES. Epidemiology standardization project (American thoracic Society). Am Rev Respir Dis 1978;118:1-120.

13 Kaye R, Chang CWD, Kazahaya K, et al. COVID-19 anosmia reporting tool: initial findings. Otolaryngol Head Neck Surg 2020;163:132-4.

14 Kazui H, Watamori TS, Honda R. [The validation of a Japanese version of the Everyday Memory Checklist]. No To Shinkei 2003;55:317-25.

15 Ware J, Kosinski M, Dewey JS. How to score and interpret singleitem health status measures: a manual for users of the SF-8 health survey. Lincoln, RI, USA, 2001.

16 Brooks R. EuroQol: the current state of play. Health Policy 1996;37:53-72.

17 Zigmond AS, Snaith RP. The hospital anxiety and depression scale. Acta Psychiatr Scand 1983;67:361-70.

18 Buysse DJ, Reynolds CF, Monk TH, et al. The Pittsburgh sleep quality index: a new instrument for psychiatric practice and research. Psychiatry Res 1989;28:193-213.

19 Kessler RC, Barber C, Beck A, et al. The world Health organization health and work performance questionnaire (HPQ). J Occup Environ Med 2003;45:156-74.

20 Satici B, Gocet-Tekin E, Deniz ME, et al. Adaptation of the fear of COVID-19 scale: its association with psychological distress and life satisfaction in turkey. Int J Ment Health Addict 2020:1-9.
21 Oken MM, Creech RH, Tormey DC, et al. Toxicity and response criteria of the eastern cooperative Oncology Group. Am J Clin Oncol 1982;5:649-56

22 Meyerowitz EA, Richterman A, Gandhi RT, et al. Transmission of SARS-CoV-2: a review of viral, host, and environmental factors. Ann Intern Med 2021:174:69-79.

23 National Institute of Infectious Diseases. Information related to coronavirus disease 2019 (COVID-19). Available: https://www.niid. go.jp/niid/ja/diseases/ka/corona-virus/2019-ncov.html

24 Li X, Xu S, Yu M, et al. Risk factors for severity and mortality in adult COVID-19 inpatients in Wuhan. J Allergy Clin Immunol 2020:146:110-8

25 Mo X, Jian W, Su Z, et al. Abnormal pulmonary function in COVID-19 patients at time of hospital discharge. Eur Respir $J$ 2020;55:2001217.

26 Bestall JC, Paul EA, Garrod R, et al. Usefulness of the medical Research Council (MRC) dyspnoea scale as a measure of disability in patients with chronic obstructive pulmonary disease. Thorax 1999;54:581-6.

27 Nishimura K, Izumi T, Tsukino M, et al. Dyspnea is a better predictor of 5 -year survival than airway obstruction in patients with COPD. Chest 2002;121:1434-40.

28 Santus P, Tursi F, Croce G, et al. Changes in quality of life and dyspnoea after hospitalization in COVID-19 patients discharged at home. Multidiscip Respir Med 2020;15:713.

29 van der Sar-van der Brugge S, Talman S, Boonman-de Winter L, et al. Pulmonary function and health-related quality of life after COVID-19 pneumonia. Respir Med 2021:176:106272.

30 Garrigues E, Janvier P, Kherabi Y, et al. Post-Discharge persistent symptoms and health-related quality of life after hospitalization for COVID-19. J Infect 2020;81:e4-6.

31 Liu K, Zhang W, Yang Y, et al. Respiratory rehabilitation in elderly patients with COVID-19: a randomized controlled study. Complement Ther Clin Pract 2020;39:101166.

32 Hawkins RB, Charles EJ, Mehaffey JH. Socio-Economic status and COVID-19-related cases and fatalities. Public Health 2020;189:129-34.

33 Arab-Zozani M, Hashemi F, Safari $\mathrm{H}$, et al. Health-Related quality of life and its associated factors in COVID-19 patients. Osong Public Health Res Perspect 2020;11:296-302.

34 Wang $\mathrm{H}$, Huang $\mathrm{D}$, Huang $\mathrm{H}$, et al. The psychological impact of COVID-19 pandemic on medical staff in Guangdong, China: a crosssectional study. Psychol Med 2020:1-9.

35 Özdin S, Bayrak Özdin Sükriye. Levels and predictors of anxiety, depression and health anxiety during COVID-19 pandemic in Turkish Society: the importance of gender. Int J Soc Psychiatry 2020;66:504-11.

36 Tomasoni D, Bai F, Castoldi R, et al. Anxiety and depression symptoms after virological clearance of COVID-19: a cross-sectional study in Milan, Italy. J Med Virol 2021;93:1175-9.

$37 \mathrm{Ji}$ D, Ji Y-J, Duan X-Z, et al. Prevalence of psychological symptoms among Ebola survivors and healthcare workers during the 20142015 Ebola outbreak in Sierra Leone: a cross-sectional study. Oncotarget 2017:8:12784-91.

38 Jiang Z, Zhu P, Wang L, et al. Psychological distress and sleep quality of COVID-19 patients in Wuhan, a lockdown City as the epicenter of COVID-19. J Psychiatr Res 2021;136:595-602.

39 Xiao H, Zhang Y, Kong D, et al. The effects of social support on sleep quality of medical staff treating patients with coronavirus disease 2019 (COVID-19) in January and February 2020 in China. Med Sci Monit 2020;26:e923549.

40 Suzuki T, Miyaki K, Song Y, et al. Relationship between sickness presenteeism (WHO-HPQ) with depression and sickness absence due to mental disease in a cohort of Japanese workers. J Affect Disord 2015;180:14-20.

41 Stawicki SP, Jeanmonod R, Miller AC, et al. The 2019-2020 novel coronavirus (severe acute respiratory syndrome coronavirus 2) pandemic: a joint American College of academic international Medicine-World academic Council of emergency medicine multidisciplinary COVID-19 Working group consensus paper. J Glob Infect Dis 2020:12:47-93.

42 Chang K-C, Hou W-L, Pakpour AH, et al. Psychometric testing of three COVID-19-Related scales among people with mental illness. Int J Ment Health Addict 2020:1-13.

43 Tzur Bitan D, Grossman-Giron A, Bloch Y, et al. Fear of COVID-19 scale: psychometric characteristics, reliability and validity in the Israeli population. Psychiatry Res 2020;289:113100. 